\title{
A REVERSE ENGINEERING APPROACH FOR MIGRATING DATA-INTENSIVE WEB SITES TO THE SEMANTIC WEB
}

\author{
Nenad Stojanovic ${ }^{1}$, Ljiljana Stojanovic ${ }^{2}$, Raphael Volz ${ }^{1}$ \\ ${ }^{\prime}$ AIFB Institute, Univ. of Karlsruhe,Germany, \{nst,rvo\}@aifb.uni-karslruhe.de \\ ${ }^{2}$ FZI Research Center for Information Technologies at the Univ. of Karlsruhe, Germany \\ stojanov@fzi.edu
}

\begin{abstract}
The Semantic Web is intended to enable machine understandable web content and seems to be a solution for many drawbacks of the current Web. It is based on metadata that formally describe the semantics of Web contents. In this paper we present an integrated and semi-automatic approach for generating shared-understandable metadata for dataintensive Web applications. This approach is based on mapping a given relational schema into an already existing ontology structure using a reverse engineering process. As a case study we present this style of a schema- and data-migration for our Institute web portal. The presented approach can be applied to a broad range of today's data-intensive Web sites.
\end{abstract}

Key words: Database reverse engineering, ontology

\section{INTRODUCTION}

The Semantic Web is one of today's hot keywords. It is about bringing "[...] structure to the meaningful content of Web pages, creating an environment where software agents, roaming from page to page, can readily carry out sophisticated tasks for users." [2]. In order to enable this, web sites are enhanced with metadata that provide formal semantics for Web content. The key technology involved here are ontologies. Ontologies provide consensual domain models, which are understandable to both human beings and machines as a shared conceptualisation of a specific domain that is given. Using ontologies, web content is made suitable for machine consumption, opposing to the content found today, which is primarily intended for human consumption. 
Currently people are slowly starting to build the Semantic Web, thus ontology-based metadata is being provided. This process of generating such metadata - also called semantic annotation - is mostly done by hand and therefore cumbersome and expensive. Visual annotation tools [9] make this task much easier, but even with sophisticated tools it is laborious to provide semantic annotations. Additionally, a maintenance problem arises; annotations must be consistent, must make proper reference, redundancy must be avoided and of course be maintained which results in a need for constant synchronization with their corresponding web content. However, because ontologies aim at consensual community (domain) knowledge, it is not enough to provide only formal semantics for information, but also realworld semantics allowing to link a machine processable content with the meaning for humans, based on consensual terminologies [7].

In this paper we address these problems and give a solution for an important class of those data-intensive web sites that draw their data from relational databases. These sites have moved away from static, fixed web pages to those that are dynamically generated at the time of user requests from data in relational databases. In order to migrate these web sites into the Semantic Web we have developed an approach which is based on mapping the given relational schema into an existing ontologic structure, using a reverse engineering process. Using this mapping the database content can be directly used to provide the intended semantic annotations. We also present a tool that supports this mapping in a semi-automatic manner.

The benefits of the proposed approach are manifold: The process of providing metadata is automated and thus inexpensive and fast. Consequently, the content of dynamic web pages is machine-understandable and therefore visible for specialized search engines. Moreover, the problem of dynamic updating metadata according to changes in corresponding web pages is also resolved. The most important benefit is that information from various community members could be exchanged on the semantic basis.

Our approach can be applied to a broad range of today's data-intensive Web sites. One of the most common applications for such data-intensive web sites are most e-commerce applications, many kinds of directories and "reporting sites"17. Such data-intensive web sites have numerous benefits, i.e. a simplified maintenance of the web design (due to complete separation between data and layout), the automated updating of web content, etc. Moreover, the using of ontologies as a mediation level for product data exchange is already proposed [15]. Our approach could be a mechanism for resolving semantic problems that arise in this integration process. 
The paper is organized as follows: Section 2 details the mapping architecture, mapping process and rules used in it. In Section 3 we present our case study by concluding with some lessons we learned. Before we conclude we contrast our contribution with related work.

\section{THE MAPPING ARCHITECTURE AND PROCESS}

\subsection{Design rationale}

Source data: Our mapping architecture is grounded on the logical database model found in running database systems [1]. The reader may note that the logical database model does not specify formal semantics of the contained information and thus is not sufficient per se as a conceptual backbone for the Semantic Web.

The reason for basing our approach on the logical data model is that most often no conceptual model (like an ER model) was created during the conception of the database or this information is lost by now. Naturally a mapping from ER models to ontologies preserves more information (like cardinalities).

The target data model for our mapping approach is F-Logic. F-Logic was developed to combine the rich data-modelling primitives of object-oriented databases with logical languages as developed for deductive databases [8].

Implementation constraints: The architecture should require only minor changes on the existing web application. Therefore the initial data is not converted. The newly implemented "Semantic Web" part of the web application should reference this source data and create the required information (in form of RDF data) on demand and dynamically by applying mapping rules specified in this paper. Another reason not to convert the data is that permanent synchronization would be needed.

Information preservation: We reinstate that the goal of this mapping is to preserve a maximum of information under the ontology framework. It is important to say that this process of the schema transformation cannot be lossless. Due to the static nature of ontologies no dynamical aspects in SQLDDLs can be converted, thus triggers, referential actions (like ON UPDATE etc.) and assertions cannot be mapped.

\subsection{Migration architecture}

The general migration architecture is depicted in figure 1 . The input of the migration is a relational model that is derived from the SQL DDL. The database schema is mapped into the given ontology using the mapping process described bellow, which applies the rules specified in the following 
sections. The same holds for database instances that are transformed into a knowledge base, which is based on the domain ontology.

The actual mapping is computed once (under the supervision and revision of the designer) and must be recomputed only if the database schema or ontology is changed. The knowledge base is computed on demand. The ontology, into which the database is mapped, is given in F-Logic. In order to publish data on the Web, F-Logic files have to be translated into RDF. This translation is straightforward. Servlets are used to create the RDF output files from the ontology and the database data. The legacy HTML files must be changed minimally to contain a reference to their metadata descriptions ${ }^{18}$. This provides the semantic annotations for the legacy content.

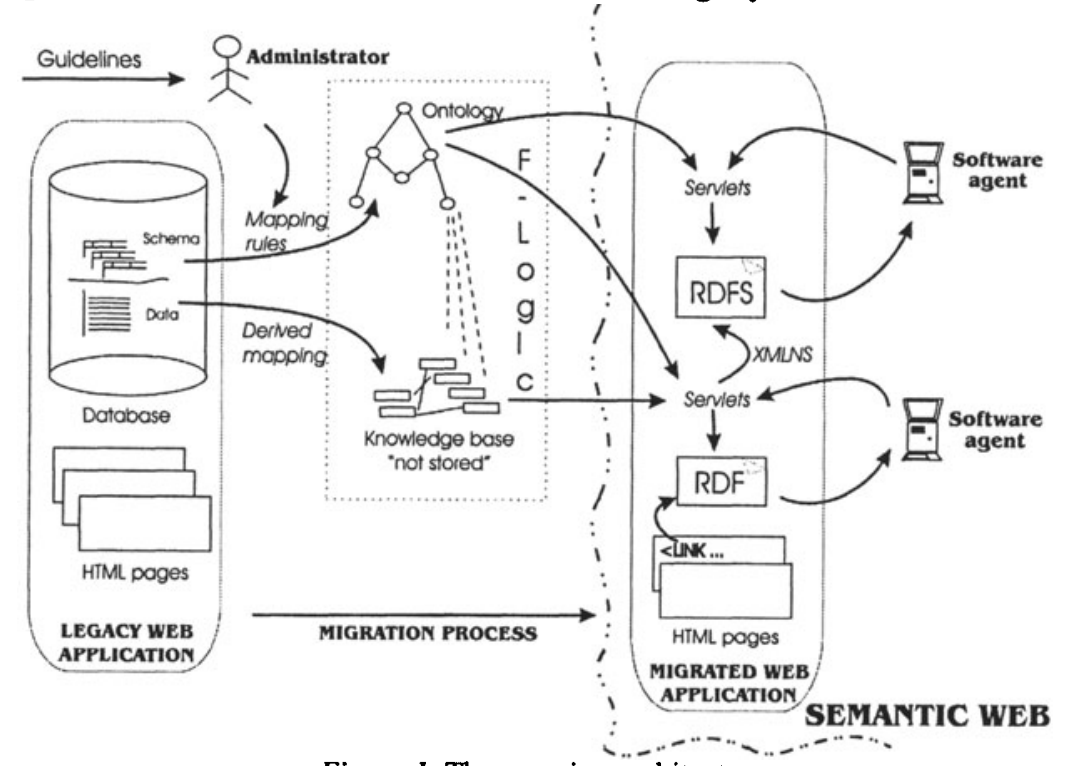

Figure 1. The mapping architecture

\section{3}

\section{Mapping Process}

The mapping process enhances the semantics of the database by providing additional ontological entities. For example, for some database entities some explicit axioms, which formally describe some properties of those entities, are added in the destination ontology (see examples given subsequently).

Our proposed mapping method consists of four steps:

1. Capture information from a relational schema through reverse engineering (consider relations, attributes, attributes types, primary keys, foreign keys / inclusion dependencies);

\footnotetext{
${ }^{18}$ HTML handles this situation with the following tag: <LINK rel=" meta"

href $=$ "mydocMetadata. DC . RDF" >
} 
2. Analyse the obtained information to map database entities into ontological entities, by considering a set of mapping rules which are briefly sketched in section 2.4 and fully described in [14]. These rules are originally made in order to enable the derivation of a new ontology from a given database schema. In this approach they are reused and adapted in order to guideline the mapping of a database schema into the given ontology (some of the remarks are given in the section 3.1). This phase is split into:

2a. alignment of the top-level terms (decision on which relation identifier corresponds to which concept identifier);

$2 \mathrm{~b}$. using existing concept creation rules to determine the relations in the relational schema that are related to a concept;

2c. using attribute creation rules to assign relation's attributes to the attributes of a concept;

Note: Since the ontology is already given, the usage of the mentioned rules does not impose changes in the definition of the ontology. These rules help in the alignment between relational entities and ontological entities.

3. Evaluate, validate and refine the mapping. Check whether all relational entities are mapped into corresponding ontological entities, i.e. whether the existing ontology is conceptually rich enough to completely represent the relational schema. Also, the implicit semantic of the relational model must be mapped into explicit ontological structures (e.g. for each $\mathrm{n}: \mathrm{m}$ relation in the relational model a rule specifies that the corresponding ontological relations are inverse).

4. Form a knowledge base ("data migration").

The implemented system, presented in next section, provides assistance in all phases. Actually, the reverse engineering process cannot be completely automated as some situations can arise where several rules could be applied. User interaction is then necessary if such ambiguities occur and domain semantics cannot be inferred.

\subsection{Mapping rules}

In [14] we have defined a set of mapping rules, which enable incremental creation of a new ontology. In this section we briefly reinterpret these rules. We refer to an example schema that models some aspects of a university. The example is depicted in figure 2 . Table 1 shows the translated schema in FLogic.

Rules for concepts: The mapping from relations to concepts is not a trivial process. We can neither assume that every concept is derived from exactly one relation nor vice versa. 
Table 1. Created ontology

\begin{tabular}{|c|c|c|}
\hline Concept & Relation & Predicate \& Axiom \\
\hline $\begin{array}{l}\text { Object[]. } \\
\text { Student::Object. } \\
\text { PhDStudent::Student. } \\
\text { School::Object. } \\
\text { Staff::Object. } \\
\text { Course::Object. } \\
\text { CourseTermin::Object. } \\
\text { CourseMaterial::Object. } \\
\text { Quarter::Object. } \\
\text { Offering::Object. }\end{array}$ & $\begin{array}{l}\text { Student[ } \\
\text { studID } \Rightarrow>\text { Number; } \\
\text { givenname }=>>\text { String; } \\
\text { familyname }=>>\text { String; } \\
\text { schoollD }>>\text { School; } \\
\text { courselD }=>>\text { Course] } \\
\text { PhDStudent[ } \\
\text { year } \Rightarrow>>\text { String]. } \\
\text { School[ } \\
\text { faculty } \Rightarrow>>\text { String; } \\
\text { studID } \Rightarrow>>\text { Student } \\
\ldots \\
\text { ]. }\end{array}$ & 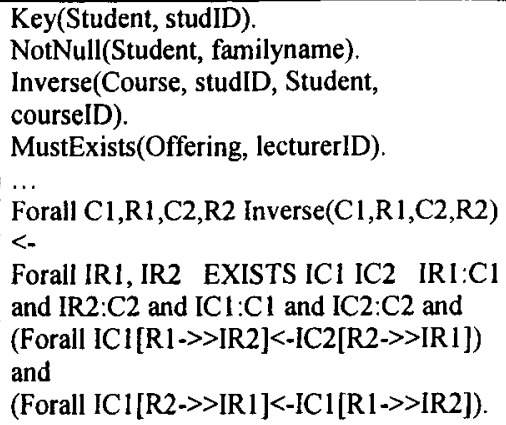 \\
\hline
\end{tabular}

The simplest way to create a concept is a direct mapping a relation from the source schema to a target concept in the ontology. However, there are two exceptions defined in two additional rules. The first of them treats relations which only exist to express $(n: m)$ relationships between some other relations. This kind of relations contains only a pair of foreign-key attributes (e.g. the relation ENROL in figure 2) and it is not converted into a concept. A second exception are those relations whose primary key is also a foreign key (e.g. the relation STAFF-DETAIL in figure 2). Most likely this relation provides additional information to the relation, which is refered to by the foreign key. Thus, this relation is not mapped into the concept, because the distributed information can be integrated into one concept.

\author{
Relational schema \\ STUDENT (studID, givenname, familyname, schoolID) \\ PhDSTUDENT (studid, year) \\ SCHOOL (schooliD; faculty) \\ STAFF (lecturerID, room) \\ STAFF-DETAIL (lecturerID, address) \\ COURSE (courseID, subject, equip) \\ COURSE-TERMIN (courscID, tnumberID, date) \\ COURSE-MATERIAL (courselD, mnumberlD, version) \\ ENROL (courseID, studID) \\ QUARTR (quarterID, year) \\ OFFERING (lecturerID, courselD, quarterID)
}

Figure 2. Relational example schema

In our example, the ENROL relation does not have a corresponding concept, as its definition contains only primary keys (courseID and studID) which are also foreign keys to the relations COURSE and STUDENT. This information is expressed by ontological relations between the concepts Course and Staff.

Moreover, no concept was derived from the relation STAFF-DETAILS, since information about a particular entity (Staff) can be collected from 
multiple relations (STAFF and STAFF-DETAILS). All other relations in example schema are converted into corresponding concepts, e.g. the relation STUDENT is mapped into a single concept Student.

Rules for inheritance: Extracting inheritance relationship from a relation schema usually requires behavioural information. It can be created when an inclusion dependency between keys of two relations exists. If for each tuple in relation $\mathrm{r} 1$ exists a tuple with the same primary key in another relation $\mathrm{r} 2$, then this can be regarded as a specialisation because attributes of both $\mathrm{rl}$ and $\mathrm{r} 2$ belong to the primary key in a tuple $\mathrm{r} 1$. For example, an inheritance relation between concept Student and concept PhDStudent is detected, based on the inclusion dependency defined on key attributes of the corresponding relations (STUDENT and PhDSTUDENT).

This rule is a specialization of the second exception for concept creation. In general the user has to decide which rule is to be applied.

Rules for ontological relations: There are several possibilities to create ontological relations from a relational schema. Again we have a default rule that is applied if no other relation rules can be applied. In this case all source attributes are converted to ontological relations. For example, the attribute givenname in the relation STUDENT is mapped to the ontological relation givenname, that applies to the concept Student.

A second relation creation rule treats many-to-many relationship. It applies if a source relation contains exactly two foreign keys and has no other attributes. This relation is then converted to multi-valued concept references. For example, the relation ENROL contains only foreign keys, referencing the STUDENT and COURSE relations. The rule leads to the creation of two multi-valued relations studID and courseID, which are attached to the corresponding concepts. These two ontological relations are mutually inverse which is additionally represented with a corresponding ontology axiom (Inverse(Course, studID, Student, courseID)).

A third rule treats one to many relationships. Again key references are converted to concept references. In distinction to previous rule on the "one" side only an ontological relation with a single-valued concept reference is created. For example, the relation STUDENT has a foreign key schoolID which refers to the primary key of the relation SCHOOL. A single-valued relation schoolID of type School, is attached to the Student concept. A multi-valued relation studID is attached to the concept School. We assume that ontological relations are multi-valued. If one ontological relation has to be single-value, the corresponding axiom (SingleValue(Student, schoolID)) is used to provide support for this type of constraints.A fifth rule groups the attributes which are distributed in several relations. All attributes (except primary keys) of the relation STAFF-DETAILS (address) are mapped into ontological relations with the domain Staff, because the relation STAFF- 
DETAILS represents additional information about the same person, which is represented by a tuple in the STAFF relation.

An inclusion dependency between the keys of two relations in both directions leads to a one-to-one relationship. We also introduce a rule that handles this kind of relationships. In this case both foreign keys are converted to single-valued ontological relations that are mutually inverse. In our example, a relation COURSE-TERMIN has the keys courseID and tnumberID referencing the keys courseID and mnumberID of the relation COURSE-MATERIAL and vice versa. The corresponding single-valued relations are attached to the created concepts. The reader may note that these relations are mutually inverse (Inverse(Course-Termin, mnumberID, Course-Material, tnumberID)).

Non-binary relationships representing relationships of higher degrees must be transformed into further concepts and a set of the binary relations. $\mathrm{RDF}$ does not provide means for $\mathrm{n}$-ary relations, therefore complex relations are decomposed into a group of several binary relations. For example, the relation OFFERING denotes a relationship between staff, courses, and quarters. For each involved database relation a new relation is added to the concept Offering connecting the corresponding concepts (Staff, Course and Quarter), which are also connect with the concept Offering. These relations are mutually inverse and single-valued. We need further semantics for this kind of relations, which ensures that the group of binary relations only exists as a whole. For example, one of the staff $S$ has offered course $C$ in quarter $Q$ if and only if there exists a course-offering $O$, such that this course-offering $O$ has relations with staff $S$, course $C$ and quarter $Q$. In order to define this, we introduce a new axiom MustExist.

\subsection{Data Migration}

Once the ontology is created, the process of data migration can start. The objective of this task is the creation of ontologicy instances (that form a knowledge base) based on the tuples of the relation database.

The data migration process has to be performed in two phases:

1. In the first phase the instances are created. A unique identifier to each instance is assigned. This translates all attributes, except for foreign-key attributes, which are not needed in the metadata.

2. In the second phase, relations between instances are established using the information contained in the foreign keys in the database tuples. This is accomplished using a mapping function, that maps keys to ontological identifiers.

In our particular implementation the Ontobroker F-Logic inference engine [5] is used. Ontobroker's API function dbaccess enables the generation of the 
instances from the given relational database on the fly. In the next section we describe a tool-support for the (semi-)automation of the presented mapping process and its application in a real-world case-study.

\section{THE AIFBNET CASE STUDY}

Our case study concerns the Web site of our Institute AIFB ${ }^{19}$, which in the first version was a database application. In order to enable the processing of the site content by machine agents we decided to describe the content explicitly and formally. However, to make the new application more sharable (for the research community) we decided to create a domain ontology - the benefits of a such approach for information integration are obvious [18].

This ontology is developed independently from the relational model of our database application, as it should sublimate community demands and not only our particular instantiation.

The ontology is developed using the OTK methodology, the process and accumulated experiences in its creation are described in [18].

Our DB-based application contains real data about researchers, projects and the institute. In order to avoid redundant work we need to map the content of the database into the knowledge base of the ontology-based Website. To do the mapping extensive tool support has to be given $t$ to give the required user interaction for the resolution of the mentioned ambiguities in the applicability of rules.

For the automation of the mapping process we used ONTOMATREVERSE, a tool for semi-automatically connecting relational databases to ontologies, implemented as a plug-in for the $\mathrm{KAON}^{20}$ ontology environment. ONTOMAT-REVERSE supports some phases in the adapted mapping process, particularly capturing information from the relational schema, validation of the mapping process and data migration. For step 2 of the mapping process ONTOMAT-REVERSE generates some recommendations based on the mapping rules [14], as will be presented in the following text.

The alignment of the top level terms is very difficult and can not be completely automated. Some guidelines could be proposed comparing entities names on the syntax level [12]. In this case that the ontology definition contains a lexical layer [13] (labels, synonyms, stems) to support a more efficient string matching performance. ONTOMAT-REVERSE supports the alignment of top level terms using the lexical layer of the AIFB ontology. For example, the source relation "Projekt" is mapped into the concept "Project", since the edit distance between "Project" is "Projekt" is

\footnotetext{
${ }^{19}$ www.aifb.uni-karlsruhe.de

${ }^{20} \mathrm{http}$ ://kaon.semanticweb.org
} 
very small. The output of this phase is a set of the mapping pairs in the form $\{$ (name_of_relation, name_of_concept) $\}$.

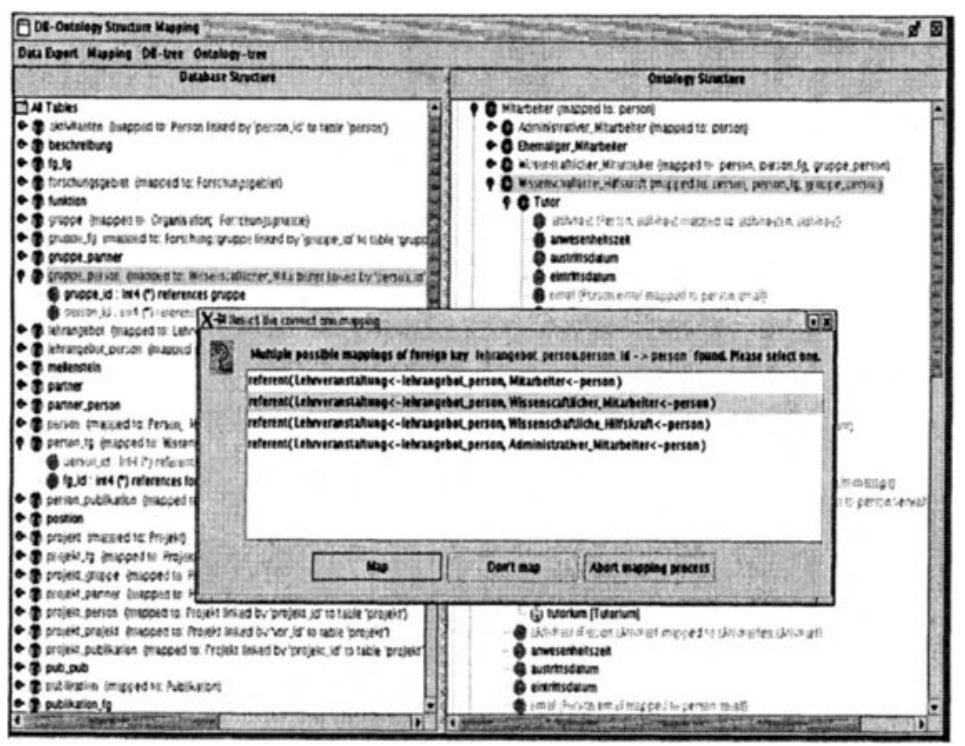

Figure 3. A screenshot of ONTOMAT-REVERSE

As already mentioned, ONTOMAT-REVERSE supports the validation step: for example, it is discovered that the relation "Beschreibung" is not mapped into any concept. After the analysis of the structure and the content of this relation, it is determined that this relation has neither foreign keys nor that other relations have a foreign key to this relation. However, the relations to the other relations are hard-coded and can be discovered only by an analysis of the content of this relation. Therefore additional heuristic rules for concept and attribute creation are implemented, which operate on the identifiers of source relations. Thereby the set of mapping pairs can be extended. For example, we can discover that the relations "Projekt_FG", "Projekt_Person", "Projekt_Gruppe" all correspond to the concept "Projekt".

Figure 3 shows how ONTOMAT-REVERSE makes recommendations for assigning attributes of the source relation to ontological relations between concepts in the ontology. The left side of figure 3 depicts the structure of the source schema. The right side shows the target ontology. Highlighted entities are mapped to each other. Recommendations for mapping attributes are listed in the dialog.

The data migration process is performed according to the guidelines described in the section 2. First object identifiers for instances are created using functions that map tuple identifiers from the source database to instance identifiers in the knowledge base. Afterwards relations between 
instances are instantiated. An illustration of the data migration process and the mentioned phases is presented in the Figure 4.

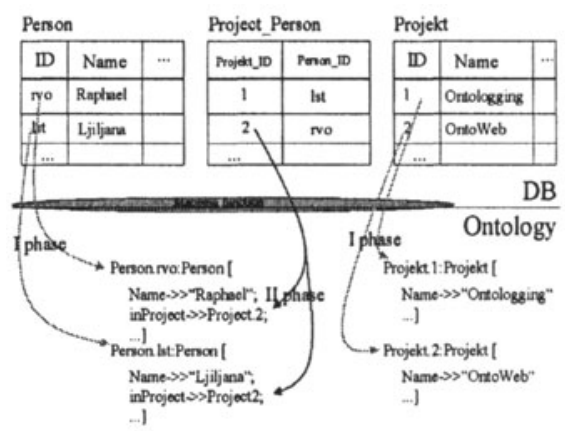

Figure 4. The data migration process - An example

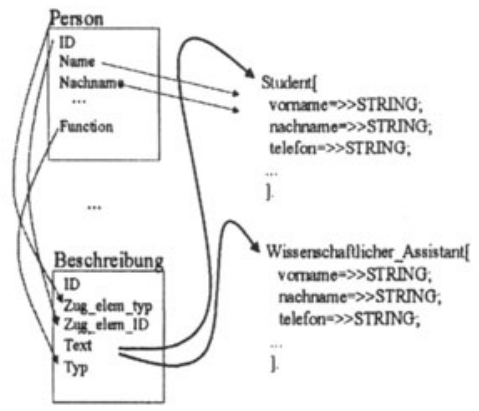

Figure 5. Type of the concept Person is codified in the attribute Function in the relation Person, but in the ontology there exists is-a hierarchy of concept Person and therefore another mapping is possible

\subsection{Lessons learned}

The result of applying our mapping process in real-world case studies suggest the formation of heuristic-mapping rules, which will be implemented in the next version of ONTOMAT-REVERSE.

- The order of applying the mapping rules is very important. Our experience suggests to create concepts first, establish the concept hierarchy afterwards and do the creation of ontological relations as a last step.

- As already emphasised is the section 2, the reverse engineering process cannot be completely automated. User interaction is necessary to resolve ambiguities and to infer domain semantics.

- - One exception for the creation of an ontological relation can be the existence of a local key in the relation schema that is used to maintain internal consistency of the data across various tables. The user might choose not to represent such local keys, which were created for implementation purposes only, in the domain ontology.

- In the case of NULL values the mapping procedure should not assign any value to the corresponding ontological relation.

- Various "irregular situations" might occur in the structure of the relational schema. For example, a relation might not have primary key. Thus IDs must be generated and the consistency of data is very hard to achieve. An example is depicted in the Figure 5. Such kind of the problems is not treated in the presented mapping approach and must be resolved per hand presenting a challenge for our future work. 
The presented user scenario could be generalized for each community. The only prerequisite is that a commonly shared community ontology exists. In the absence of the community ontology, our mapping process [17] can be used for generating an ontology from the existing relational schema, which can then form a basis for a shared agreement in the given community.

\section{RELATED WORK}

As known to the authors, there is no approach that integrates aspects of reverse engineering and integration platforms for the metadata on the (Semantic) Web, so those topics will be discussed separately.

Database reverse engineering: There are very few approaches investigating the transformation of a relational model into an ontological model. The most similar approach to our approach is the project Infosleuth [11]. In this project an ontology is built based on the database schemas of the sources that should be accessed. The ontology is refined based on user queries. However, there are no techniques for creating axioms, which are a very important part of an ontology. Our approach is heavily based on the mapping of some database constraints into ontological axioms. Moreover, the semantic characteristics of the database schema are not always analysed.

More work has been addressed on the issue of explicitly defining semantics in database schemas [4], [16], extracting semantics out of database schema [4], [10] and transforming a relational model into an object-oriented model [3], which is close to an ontological theory. Rishe [16] introduces semantics of the database as a "means" to closely capture the meaning of user information and to provide a concise, high-level description of that information. In [4] an interactive schema migration environment that provides a set of alternative schema mapping rules is proposed. In this approach, which is similar to our approach on the conceptual level, the reengineer repeatedly chooses an adequate mapping rule for each schema artefact. However, this stepwise process creates an object-oriented schema, therefore axioms are not discussed.

Information integration: In the broader sense our approach could be treated as an information integration approach [14], [19], while we provide a platform for resolving semantic problems that arise in this integration process. The information mediation architecture contains intermediate layer that mediates between human user and information sources. That layer has a dual structure: the mediator deals with the human user and the wrappers deal with the information sources [7]. In our approach information sources have a uniform structure, so that the role of wrapping is dedicated to the mapping process. In [6] an approach for reconciling XML data is presented, based on intermediate conceptual models. In this case, a human expert is needed to 
reverse-engineer the underlying conceptual model for an XML schema, and to specify formally how the original schema maps onto the corresponding conceptual model. Our approach provides the guidelines how to make such kind of reverse engineering, so that some phases can be automated and user intervention is avoided.

\section{CONCLUSION}

In this paper we proposed a novel, integrated and semi-automated approach for migrating data-intensive Web applications into the Semantic Web which can be applied to a broad range of today's business Web sites.

The approach starts with transforming the relational database model into corresponding ontological structures, which are then used for mapping the content of the database into an ontology-based knowledge base. The knowledge base can then be published on the web using RDF.

Publishing such statements on the web makes content of the web pages (in the last instance also the content of a given database) public and machine understandable, which is the prerequisite to achieve the Semantic Web. As a side effect, the issue of making the semantics of a database more explicit is also addressed and our approach leads to more formal semantics that could be used, for example, for the maintenance of data-driven applications and also in the simplified migration to other databases.

Moreover, the presented scenario can be very popular in the e-commerce domain, where the usage of ontologies as mediation level for product data exchange is already proposed [15] and our approach could be a mechanism for resolving the semantic problems that arise in this integration process.

The benefits of the proposed approach are manifold: The process of providing metadata is automated and thus inexpensive and fast. The problem of dynamic updating metadata according to changes in corresponding web pages is also resolved. The most important benefit is that information from various community members could be exchanged on the semantic bases. This paves the way to unleash the full-power of the Semantic Web.

\section{ACKNOWLEDGEMENTS}

The research presented in this paper would not have been possible without our colleagues and students at the Institute AIFB, University of Karlsruhe, and FZI, Karlsruhe. We thank out student Gert Pache who implemented ONTOMAT-REVERSE. Research for this paper was partially financed by EU in the IST-funded projects "OntoLogging" and "WonderWeb".

\section{REFERENCES}

1. Abiteboul S., Hull R., Vianu V. Foundation of databases, Addison-Wesley Publishing Company, 1995. 
2. Berners-Lee T., XML 2000 - Semantic Web talk, http://www.w3.org/2000/Talks/1206$\mathrm{xml2k}$-tbl/slide 10-0.html, 2000

3. Behm A., Geppert A., Dittrich, K., On the Migration of Relational Schemas and Data to Object-Oriented Database Systems, Proc. of the 5th Int. Conference on ReTechnologies for Information Systems, pp. 13-33, 1997.

4. Chiang R., Barron T., Storey V., Reverse engineering of relational databases: Extraction of an EER model from a relational database, Journ. of Data and Knowledge Engineering, Vol. 12, No. 2, March 1994, pp. 107-142., 1994.

5. Decker S., Erdmann M., Fensel D., Studer R., Ontobroker: Ontology Based Access to Distributed and Semi-Structured Information, In R. Meersman et al., editors, Database Semantics: Semantic Issues in Multimedia Systems, pps 351-369. Kluwer Academic Publisher, 1999.

6. Decker S., van Harmelen F., Broekstra J., Erdmann M., Fensel D., Horrocks I., Klein M., Melnik S., The Semantic Web - on the Roles of XML and RDF, IEEE Internet Computing. September/October, 2000.

7. Fensel D., Ontologies: Dynamic Networks of Meaning, International Semantic Web Working Symposium (SWWS), Stanford, July 2001.

8. Kifer M., Lausen G., Wu J., Logical Foundations of Object-Oriented and Frame-Based Languages, Journal ACM, 42:741-843, 1995.

9. Handschuh, S., Staab, S., Maedche, A. CREAM - Creating relational metadata with a component-based, ontology-driven annotation framework, ACM K-CAP 2001. October, Vancouver.

10. Hainaut J.L., Database Reverse Engineering, Models, Techniques and Strategies. 10th Conf. on ER Approach, San Mateo (CA), 1991.

11. Kashyap V., Design and creation of ontologies for environmental information retrieval, 12th Workshop on Knowledge Acquisition, Modelling and Management (KAW'99), Banff, Canada, October 1999.

12. Levenshtein I. V., Binary Codes capable of correcting deletions, insertions, and reversals, Cybernetics and Control Theory, 10(8):707-710, 1966.

13. Maedche A., Ontology Learning Supporting Emergent Semantics, IEEE Intelligent Systems - Trends \& Controversies, 2002.

14. Melnik S., Decker S., A Layered Approach to Information Modeling and Interoperability on the Web, In Proceedings of the Workshop "ECDL 2000 Workshop on the Semantic Web", 2000.

15. Omelayenko B. Fensel D., An Analysis of B2B Catalogue Integration problems: Content and Document Integration, In Proceedings of the International Conference on Enterprise Information Systems (ICEIS-2001), 2001.

16. Rishe N., Database Design: The Semantic Modeling Approach, McGraw-Hill, 1992.

17. Stojanovic L., Stojanovic N., Volz R., Migrating data-intensive Web Sites into the Semantic Web, ACM Symposium on Applied Computing SAC, Madrid, 2002.

18. Stojanovic N., Maedche A., Staab S., Studer S., Sure Y., SEAL - A Framework for Developing SEmantic PortALs. ACM K-CAP-2001, 2001.

19. Wache H., Vögele T., Visser U., Stuckenschmidt H., Schuster G., Neumann H., Hübner C., Ontology-Based Integration of Information - A Survey of Existing Approaches, In Proceedings of the IJCAI-01 Workshop on Ontologies and Information Sharing, pp 108118, Seattle, USA, August 4-5, 2001. 